\title{
Mizutama: A Quick, Easy, and Accurate Method for Counting Erythrocytes
}

\author{
David Ochoa ${ }^{1}$ \\ Tomás Redondo ${ }^{1}$ \\ Gregorio Moreno-Rueda ${ }^{2, *}$ \\ ${ }^{1}$ Departamento de Etología y Conservación de la \\ Biodiversidad, Estación Biológica de Doñana, Consejo \\ Superior de Investigaciones Científicas, Avenida Américo \\ Vespucio s/n, 41092 Sevilla, Spain; ${ }^{2}$ Departamento de \\ Zoología, Universidad de Granada, E-18071 Granada, Spain
}

Accepted 10/31/2018; Electronically Published 2/7/2019

Online enhancement: supplemental table. Dryad data: https:// dx.doi.org/10.5061/dryad.bk2690n.

\begin{abstract}
Hematological profiles are routinely used to assess the health status of animals. Several methods have been developed for blood cell counting, but they are typically expensive and/or time-consuming. Here, we present a free image-processing software, Mizutama, developed for counting cells in photographs of blood smears. Mizutama uses the thresholding method to transform original photographs into grayscale trinary images. Following a number of parameters, Mizutama searches in the image for cells of a given size, with a nucleus size relative to cytoplasm surface area. The software is not only easy, versatile, and intuitive to handle but also fast when counting cells in photographs. Moreover, we show that it is highly accurate, failing to detect only ca. $1.4 \%$ of avian red cells in ordinary microscopic photographs. The Mizutama application may greatly facilitate the counting of erythrocytes and other blood cells in physiological studies, saving time and money.
\end{abstract}

Keywords: hematology, erythrocytes, leukocytes, image-processing software, health status.

*Corresponding author; email: gmr@ugr.es.

Physiological and Biochemical Zoology 92(2):206-210. 2019. (C) 2019 by The University of Chicago. All rights reserved. 1522-2152/2019/9202-8076\$15.00. DOI: $10.1086 / 702666$

\section{Introduction}

Hematological parameters provide valuable information on health and immune performance, and blood cell profiles are routinely used in physiological studies (Campbell and Ellis 2013). For hematological profiles, erythrocyte counting is usually necessary to express leukocyte profiles relative to number of erythrocytes. Moreover, erythrocyte counting is also basic in studies of hemoparasites, which are quantified per number of erythrocytes (Godfrey et al. 1987).

Several methods have been developed for blood cell counting with different advantages and disadvantages (Brown et al. 1993). Automated devices, such as flow cytometry, may quickly classify and count different blood cell types (Brown and Wittwer 2000). However, these devices are usually expensive, require considerable training to use, and need fresh blood, hindering their use with blood from field studies. The most frequently used methods for cell counting involve hemocytometers (Walberg 2001), which are far cheaper and easier to use than flow cytometers. However, these devices also require fresh blood, hampering their application in field studies, and show a relative large error $(20 \%-30 \%$; Russo et al. 1986). The cheapest and easiest method, especially in field studies, is to estimate the cell count from stained blood smears (Campbell and Ellis 2013). Moreover, this method requires only a drop of blood, and the blood sample may be preserved for future studies or reexamination. The main drawback of this method is that it is highly time-consuming, especially because leukocyte or hemoparasite profiles require thousands of erythrocytes to be visually counted to ensure a good estimate. For greater speed, Gering and Atkinson (2004) developed a protocol to count erythrocytes in blood-smear photographs by using ImageJ software. This protocol recognizes cell nuclei according to a given size, and erythrocyte counting takes only 3 min per photograph, signifying considerable time savings.

Here, we present a free image-processing software application, Mizutama, developed for counting cells in microscopic blood-smear photographs. This software is not only easy and intuitive to handle but also very fast, solving the main disadvantage of visual counting. Moreover, although initially designed for counting avian erythrocytes, its versatility to distinguish cell types according to contrast, overall size, and relative size of nucleus versus cytoplasm makes the software useful for also detecting leukocytes in photographs. Our software functions in a way similar to the method developed by Gering and Atkinson (2004) but shows a number of advantages: it is easy to handle for users not familiar with ImageJ, shows broader versatility to detect different cell 
types, and, most importantly, is faster in processing photographs, taking only a few seconds per photo.

\section{Functioning of Mizutama Software}

Mizutama is a Delphi 2.0 (Manning 1996) application. The application, source code, and user manual are deposited in the Dryad Digital Repository (https://dx.doi.org/10.5061/dryad.bk2690n; Ochoa et al. 2019). This image-processing software was designed to count cells in ordinary microscopic photographs (maximum of 3,600 pixels allowed in JPG or JPEG format) of blood smears. It uses the thresholding method (see Shapiro and Stockman 2002) to transform original photographs into grayscale trinary images, where pixels are categorized as positive, gray, or null, according to a specified threshold criterion. In the case of avian erythrocytes, Mizutama searches photographs for spots (the nucleus of the erythrocyte) that may be surrounded by a lighter halo (the cytoplasm). Mizutama identifies these elements according to the parameters specified and counts their number. The search and identification is based on five parameters that must be specified in the program. (1) Contrast (button Contraste) determines how dark a pixel must be in order to be considered positive. It could have any positive value, allowing the software to be used with photographs varying in the degree of staining or illumination. Moreover, if different cell types vary in saturation, the contrast value can be adjusted to facilitate their discrimination. (2) Gray threshold (button Umb. Gris) determines how dark a pixel must be in order to be considered gray. Values range between 0 (completely white) and 1 (as dark as positive pixels). With these two parameters, Mizutama classifies the pixels according to their light intensity (I). For $\mathrm{I}<$ contrast, the pixel is considered positive; for contrast $<\mathrm{I}<$ gray threshold, the pixel is considered gray. For I $>$ gray threshold, the pixel is considered null. (3) Diameter (button Diámetro) sets the size of the circle equivalent to the cell (i.e., the size of the cell in the photograph). This should be a positive value based on the size of the cell to be counted and the microscope magnification used. With this parameter, the software scans the image, searching for positive pixels, and when a positive pixel is found, a circle of the size previously set is superimposed over the pixel.

The next two parameters determine whether the spot found can be considered the cell type being sought. (4) Fraction (button Fracción) indicates the proportion of positive pixels that must be found inside the circle in order to be considered a nucleus (i.e., the relative size of the nucleus). A value of 1 indicates that half of the pixels inside a circle correspond to positive values (i.e., the nucleus surface area is half the cell surface area). (5) Gray fraction (button Fr. Gris) indicates the minimal proportion of gray pixels that must be found inside the specified diameter in order to be considered a halo and therefore to count the spot as a cell (i.e., the relative size of the cytoplasm).

With these latter two parameters, we determine the relative size of the nucleus and cytoplasm of the cell. Therefore, the software can distinguish cells that differ in size or in the relation of nucleus surface area versus cytoplasm surface area. For example, to avoid counting leukocytes as erythrocytes, given that cytoplasm is typically smaller in leukocytes than in erythrocytes, we can in- crease the value of the gray fraction so that only cells having a relatively large cytoplasm are counted (fig. 1). This would exclude leukocytes from the counting. By contrast, to count only leukocytes, we can indicate a relatively large nucleus (fraction $=0.8$ ) but small cytoplasm (gray fraction $=0.1$; fig. $1 C$ ).

When the software is run, we can select only one photograph (with the button Uno) or analyze a set of photographs stored in a folder (with the button Lista). In both cases, an explorer window enables us to select the file or folder we wish to analyze. Once the file to analyze is selected, the software identifies a cell according to the procedure described above. Next, it shows a grayscale image of the photograph that classifies the pixels (black, gray, or white), highlighting the identified spots (fig. 1). In red, the software surrounds the spots that match the parameters that were set, identifying the cells being searched for. Then, Mizutama counts the number of red circles in the image and gives the values in the upper bar of the window or, when in batch mode, in a TXT file. For each photograph, the output includes the values set for contrast, diameter, and fraction (first three columns from the left); the number of positive pixels in the photograph (fourth column); the number of correct cells counted (fifth column); and information on the photograph, its size in pixels (sixth and seventh columns), and its title (last column).

The software also uses green circles to indicate any spots that fulfill the parameters for the nucleus (fraction) but not for the cytoplasm (gray fraction); these spots may represent a cell type we do not wish to count (e.g., isolated thrombocytes). Yellow circles highlight spots that do not fulfill all the parameters necessary to be considered a cell (e.g., staining spots or ruptured cells), leaving the evaluation to visual inspection.

\section{Testing the Accuracy of the Software to Count Avian Erythrocytes}

With the use of a sterile syringe and needle (30 gauge), blood samples were collected from the jugular vein of 6415 -d-old chicks (Gallus domesticus) kept at the Animal Experimentation facilities of the Doñana Biological Station. The chicks were housed in four $300 \times 50 \times 50$-cm cages $(15-19$ chicks per cage) and submitted to the husbandry protocol of Fairchild (2009): $30^{\circ}-34^{\circ} \mathrm{C}$ (according to age), light cycle of 18L:6D23L:1D (according to age), 60\% humidity, ad lib. access to food and water, floor covered with artificial lawn, and cages cleaned daily. The experimental procedure was approved by the Consejo Superior de Investigaciones Científicas Ethical Committee and licensed by the Andalusian Competent Authority according to Spanish and European legislation. One drop of blood was used per thin smear, which was air dried and fixed with methanol. Smears were stained for $40 \mathrm{~min}$ with Giemsa diluted 1:10 in buffer with pH 7.2 (Campbell and Ellis 2013). For each smear, a minimum of 35 photographs of different fields (randomly selected) at $\times 400$ magnification were taken with a microscope (Zeiss Axio Imager A1) equipped with a coupled camera (Zeiss Axiocam ERc 5s). The edges of the smears were avoided in taking the photographs as well as any 


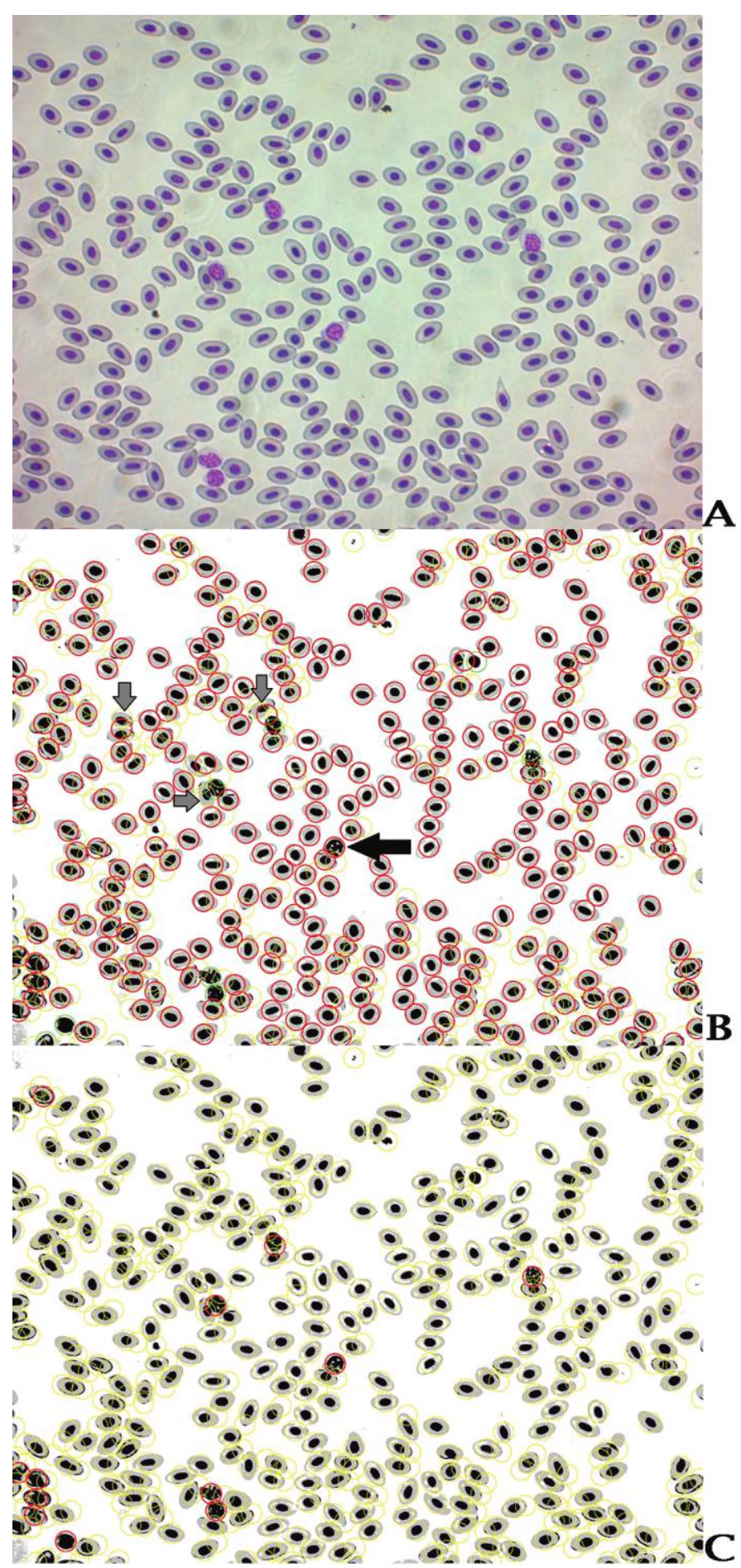

Figure 1. Original photograph $(A)$ and the same photograph after processing by the software $(B, C)$. In $B$, note that most of the leukocytes are not considered erythrocytes (only one leukocyte [black arrow] lies within a red circle) and are signaled in green. Note that several spots are signaled in yellow. Most of the erythrocytes were included in red circles, with only a few exceptions (gray arrows). In $C$, the parameters were adjusted to detect only leukocytes in the same photo.

areas showing staining or fixation problems hampering a clear view of the cells.

Before processing the photos of a sample (i.e., a smear), we randomly took three photos in order to calibrate the contrast, gray threshold, diameter, fraction, and gray fraction parameters. Parameters were calibrated by a trial and error method, inspecting the cells Mizutama had selected and adjusting the parameters to be sure that only erythrocytes (and not leukocytes) 
were counted and that all erythrocytes were correctly identified. After these parameters were set, the counting was carried out by analyzing the whole set of photos corresponding to a given smear.

To check the accuracy of the program, we randomly set aside a subsample of at least 10 images from each smear (in total, $n=680$ images), which were used to count the number of erythrocytes in each using Mizutama. These results were compared with the counts made on visual inspection by one researcher (D. Ochoa, blinded for the Mizutama results), which were considered to be the actual value. With the difference in erythrocytes counted visually and by Mizutama, we estimated the percentage of error of the software and analyzed whether the error was directional (i.e., the software systematically over- or underestimated numbers). In addition, we tested the correlation between the Mizutama value and the visually recorded one.

\section{Results}

The automated counting performed by Mizutama rendered $432.6 \pm 5.6$ (SE) erythrocytes per photograph (range: 170951), whereas the actual count performed visually was $439.8 \pm$ 5.8 erythrocytes per photograph (range: 174-964; raw data in table S1, available online). The difference between the actual value and the estimate by the software was $7.2 \pm 0.5$ erythrocytes per photograph (range: -14 to 90 ). This difference was significantly biased $\left(t_{679}=14.1, P<0.001\right)$; that is, the software tended to count fewer erythrocytes than counted visually. The error slightly increased with the number of erythrocytes in the sample $(r=0.36, P<0.001)$. Nonetheless, this error was only of $1.4 \% \pm 0.1 \%$ (range: $-5.6 \%$ to $17.4 \%$ ). In fact, the correlation between the actual value and the count by Mizutama was very strong ( $r=0.996, P<0.001$; fig. 2).

\section{Discussion}

Mizutama is a type of image-processing software designed for quick and easy cell counting in microscopic photographs of blood smears. Here, we show that the software is highly accurate counting nucleated erythrocytes, although the versatility of the software also allows the counting (or at least to facilitate detection) of other cell types, such as leukocytes (see fig. 1C). The software typically counts fewer erythrocytes than the number that actually exists, but the error is only $1.4 \%$ on average (seven erythrocytes fewer per photograph). The error increases with erythrocyte numbers, suggesting a higher bias as smears become denser. Nonetheless, erythrocyte density explained only $13 \%$ of the error variance.

The use of Mizutama to count blood cells in photographs has a number of advantages relative to the use of flow cytometry, especially the low cost and easy handling. Moreover, correlations between cell count by flow cytometry and blood smears are good (Burraco et al. 2017) but lower than between Mizutama and visually counting in blood smears. That is, visual cell counts in blood smears appear to be more accurate than counts by flow cytometers. In comparison to hemocytometers, Mizutama also provides a number of advantages: less blood needed, easier handling, sample preservation and storage, cheaper, and apparently quicker. The widespread use of the hemocytometer versus smear counting was precisely because the visual counting of erythrocytes proves prohibitively time-consuming.

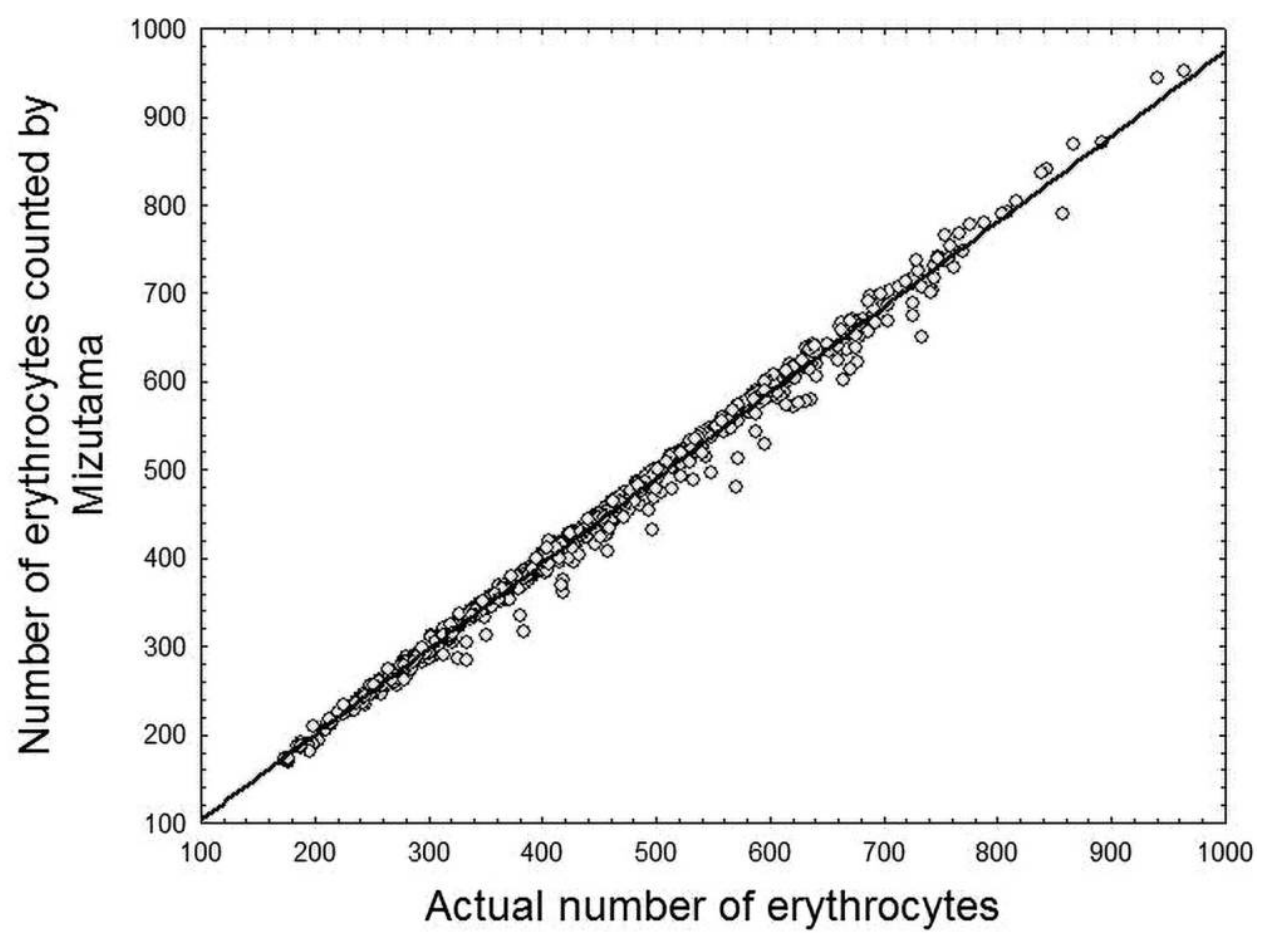

Figure 2. Correlation between actual count of erythrocytes (performed visually) and the Mizutama count of erythrocytes. 
The time invested in counting erythrocytes might be reduced by counting cells in only some fields and assuming that the density of erythrocytes is uniform throughout the remaining fields. However, in our sample, coefficients of variation within smears fluctuated between $10.4 \%$ and $45.7 \%$, with ranges in erythrocyte counts inside the same smear oscillating from 98 to 790 . These variations indicate the risk of assuming that the number of erythrocytes in a few fields are representative of the complete smear. Moreover, these values might vary with the ability of researchers to prepare homogeneous smears. In conclusion, we encourage researchers to use Mizutama, which not only allows a quick, accurate, and versatile method to count erythrocytes but also offers additional possibilities for counting other cell types, such as leukocytes.

\section{Acknowledgments}

Enrique Collado from the Doñana Biological Station Information Technology Service kindly developed the software. This work was supported by the Ministerio de Economía, Industria y Competitividad/Agencia Estatal de Investigación/Fonds Européen de Développement Régional, Union Européenne (projects CGL2011-29694, CGL2014-55969-P, and CGL2017-84938-P). Comments by two anonymous reviewers improved the manuscript. David Nesbitt improved the English.

\section{Literature Cited}

Brown B.A., R.C. Hunter, A. O’Hare, and G. Erim. 1993. Hematology: principles and procedures. Lea \& Febiger, Philadelphia.
Brown M. and C. Wittwer. 2000. Flow cytometry: principles and clinical applications in hematology. Clin Chem 46:12211229.

Burraco P., F. Miranda, A. Bertó, L.A. Vazquez, and I. GomezMestre. 2017. Validated flow cytometry allows rapid quantitative assessment of immune responses in amphibians. Amphib-Reptil 38:232-237.

Campbell T.W. and C.K. Ellis. 2013. Avian and exotic animal hematology and cytology. Blackwell, Ames, IA.

Fairchild B.D. 2009. Environmental factors to control when brooding chicks. Univ. Ga. Coop. Ext. Bull. 1287.

Gering E. and C.T. Atkinson. 2004. A rapid method for counting nucleated erythrocytes on stained blood smears by digital image analysis. L Parasitol 90:879-881.

Godfrey R.D., Jr., A.M. Fedynich, and D.B. Pence. 1987. Quantification of Hematozoa in blood smears. LWildl Dis 23:558-565.

Manning M.M. 1996. Delphi 2. Prentice Hall Hispanoamericana, Mexico.

Ochoa D., T. Redondo, and G. Moreno-Rueda. 2019. Data from: Mizutama: a quick, easy, and accurate method for counting erythrocytes. Physiol Biochem Zool, Dryad Digital Repository, https://dx.doi.org/10.5061/dryad.bk2690n.

Russo E.A., L. McEntee, L. Applegate, and J.S. Baker. 1986. Comparison of two methods for determination of white blood cell counts in macaws. LAm Vet Med Assoc 189:10131016.

Shapiro L.G. and G.C. Stockman. 2002. Computer vision. Prentice Hall, Upper Saddle River, NJ.

Walberg J. 2001. White blood cell counting techniques in birds. Semin Avian Exot Pet Med 10:72-76. 